\title{
CONTRIBUTION A L'ÉTUDE DE LA HOULE AU VOISINAGE DES COTES
}

\author{
par L. CARLOTTI \\ Ingénieur civil de l'Ecole d'Application du Génie marifime \\ Ingénieur principal des Travaux publics de la France d'outre-mer
}

(SUITE)

CHAPITRE $\|$ II

\section{COMPARAISON ENTRE LES ESSAIS EFFECTUES SUR UNE MAQUETTE CONSTRUITE SANS DISTORSION DES PROFONDEURS, ET CEUX UTILISANT LA DISTORSION}

\section{3,1 - Essais réalisant simultanément toutes les similitudes : Maquettes sans distorsion.}

L'intérêt des essais sur modèle réduit réside dans la valeur de la prédétermination des rap. ports $N$ existant entre la dénivellation provoquée par la houle incidente ou point repère à l'extérieur du port et la dénivellation en des points du port, particulièrement intéressants.

On a vu, lors de l'étude systématique des différentes causes du rapport $N$, que la similitude relative à l'épanouissement est réalisée lorsque la similitude de formes est satisfaite.

La réalisation des deux autres facteurs de la similitude d'amortisssement n'est rigoureusement réalisable que si le modèle est construit avec $\frac{\alpha}{\theta^{2}}=1$. On peut donc conclure que les maquettes construites sans distorsion des profondeurs sont parfaites, sous réserve que l'échelle soit telle que l'abaque d'où est tirée la figure 2 du paragraphe 2.22 soit encore utilisable. Cependant, les maquettes ainsi construites ayant des dimensions exagérées et étant très coûteuses, on peut se demander dans quelle mesure on pourrait se contenter d'essais exécutés avec des maquettes ayant $\frac{\alpha}{\theta^{2}}>1$, se bornant ò ne conserver rigoureusement que la similitude de formes.

\section{3,2 - Comparaison de la surface d'une maquet te construite avec la distorsion maxima, of de la surface de la maquette correspon dante construite sans distorsion.}

Les esscis sont d'autant moins coûteux que la surface de la maquette est plus réduite, donc que l'échelle $\alpha$ des dimensions horizontales est petite.

\section{Détermination de la maquette construite avec distorsion maxima.}

Nous allons faire une application numérique de la méthode exposée au Chapitre premier, paragraphe 1,2, pour chiffrer l'avantage que présente l'usage de la distorsion maxima définie par la formule 7 bis.

Considérons un port pour lequel la gamme des périodes à étudier est comprise entre:

$$
\underset{\operatorname{minima}}{T}=9 \mathrm{sec} ., 50 \text { et } \underset{\text { maxima }}{T}=12 \mathrm{sec} .50 \text {. }
$$

La période $T_{\text {" }}$ pour laquelle la maquette sera construite est : $T_{1}=11$ secondes.

Supposons que les photos aériennes montrent que l'emplacement repère se trouve dans la région de profondeur.

$$
\mathrm{H}=16 \text { mètres } 50 \text {. }
$$

L'abaque de la planche I donne

$$
\Lambda=128 \text { mètres, pour } \mathrm{H}=16 \mathrm{~m} .50
$$

et $T=11$ secondes.

La formule (7bis) donne:

$$
\frac{\alpha}{\theta^{2}}=1,5
$$

L'échelle des temps $\theta$ est choisie pour que la période $t$ correspondant à $T=9 \mathrm{sec} .5$, soit nettement au-dessus du domaine des houles capillaires; on prend : 
cela donne: $\theta=\frac{0,345}{9,5}=\frac{1}{27,5}$

L'échelle des distances horizontales est alors: $\alpha=1,5 \times\left(\frac{1}{27,5}\right)^{2}=\frac{1}{500}$

La profondeur $h_{10}$ correspondant à $\mathrm{H}=10 \mathrm{~m}$. sur le réel; tirée de la formule (5) du paragraphe 1,2 est alors : $h_{(1)}=3,700 \mathrm{~cm}$.

\section{Détermination de la maquette construite sans} distorsion.

On admet, d'une façon générale, que, pour ne pas avoir une nappe d'eau trop mince, on adopte une valeur de $h_{10}$ égale à $3 \mathrm{~cm}$.

$$
h_{10}=3 \mathrm{~cm} \text {. }
$$

Cela conduit à une échelle:

$$
\alpha=\frac{3}{1000}=\frac{1}{333}
$$

La maquette construite sans distorsion est donc à une échelle 1,5 fois plus grande que celle construite avec distorsion (dans ce cas particulier uniquement) et le rapport des surfaces (donc des prix) des deux maquettes est de 2,25.

\section{Essais avec des maquettes conservant unique- ment la similitude de formes.}

Dans ce cas, pour chaque paire de points homologues du fond réel et du modèle, on $a$ :

$$
\frac{h}{\lambda}>\frac{H}{\Lambda}
$$

On a vu que, dans ce cas, la similitude d'épa. nouissement est réalisée.

\section{3,31 - Comparaison de l'évolution de l'ampli-} tude $\eta$ en fonction de la profondeur $H$ et du chemin parcouru $x$ sur le réel et sur une maquetre réalisant uniquement la similitude de formes.

Considérons maintenant ce que devient la similitude relative aux variations de n avec $H$.

Puisqu'il s'agit de phénomènes comportant des houles en régime établi, il y aura une valeur de T pour la houle dans le port réel et une valeur correspondante $t$ de la période sur le modèle.

Cherchons la relation existant entre $U$ et $C$ d'une part, et $/ \frac{H}{\Lambda}$ d'autre part, au fur et à mesure que la houle se propage sur des fonds de profondeur décroissante.
Lo relation (16) définissant $U$ peut s'écrire:

(21) $Z=\frac{4 \pi}{g T} U=\operatorname{Th}\left(\frac{2 \pi H}{\Lambda}\right)\left(1+\frac{\left(\frac{4 \pi H}{\Lambda}\right)}{\operatorname{sh}\left(\frac{4 \pi H}{\Lambda}\right)}\right.$ et la relation (1) définissant $c$ peut s'écrire:

$$
\text { (22) } Y=\frac{2 \pi}{g T} C=T h\left(\frac{2 \pi H}{\Lambda}\right)
$$

Ces relations se traduisent par les courbes de la figure 3 ci-après.

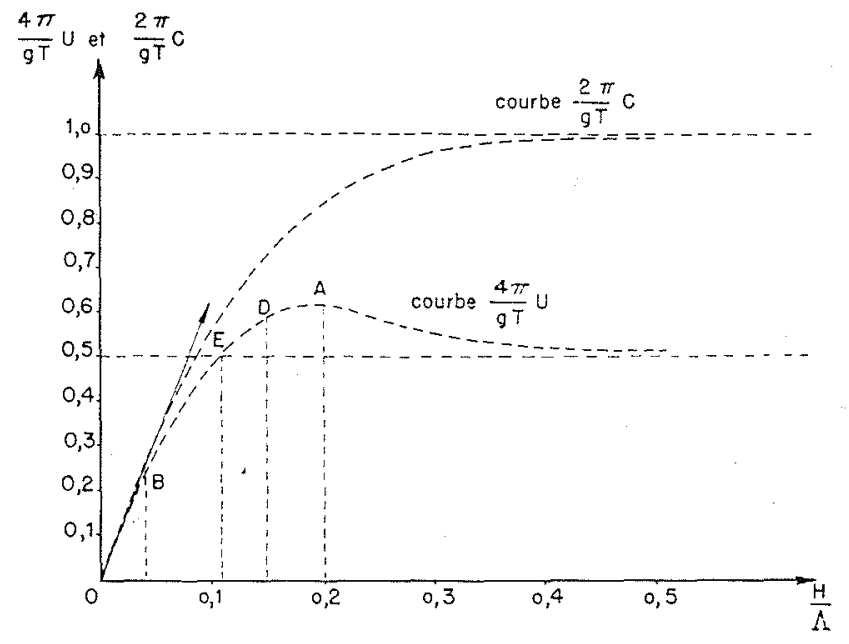

Fig. 3

La variation d'amplitude d'une crête au fur et à mesure qu'elle chemine depuis le large vers le rivage est donnée par la formule (15) qui dépend des deux fonctions :

$$
U \times \frac{4 \pi}{g T}=f_{1}\left(\frac{H}{\Lambda}\right) \text { et } \beta \Lambda=f_{2}\left(\frac{H}{\Lambda}\right)
$$

(voir figure 4)

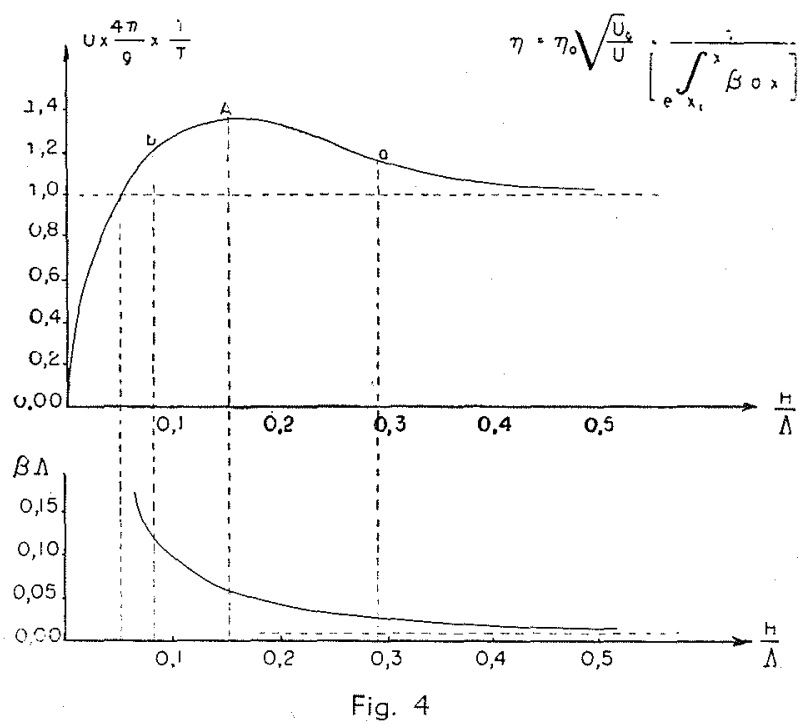


L'arc de la courbe $\left(\frac{4 \pi}{g T}\right) \cup=f_{1}\left(\frac{H}{\Lambda}\right)$ de la figure 4 qui nous intéresse, peut être schéma. tisé par les quatre points $A, D, E, B$.

Regardons, pour fixer les idées, à quelles profondeurs $H$ comptées au-dessous du niveau réel de la mer (y compris la marée) correspondent $A, D, E$ et $B$, lorsque la période de la houle incidente est :

$$
\begin{array}{ll}
T=8 \text { secondes } & \text { houles fréquentes, } \\
T=10 \text { secondes } & \text { tempêtes, } \\
T=14 \text { secondes } & \text { tempêtes exceptionnelles. }
\end{array}
$$

\begin{tabular}{|c|c|c|c|c|c|c|}
\hline & \multicolumn{2}{|c|}{$T=8 \mathrm{sec}}$. & \multicolumn{2}{|c|}{$T=10 \mathrm{sec}$} & \multicolumn{2}{|c|}{$T=14 \mathrm{sec}$} \\
\hline & $\mathrm{H}$ & $\Lambda$ & $\mathrm{H}$ & $\Lambda$ & $\mathrm{H}$ & $\Lambda$ \\
\hline Région A & $16,25 \mathrm{~m}$ & $83,5 \mathrm{~m}$ & $25,50 \mathrm{~m}$ & $131 \mathrm{~m}$. & $49,90 \mathrm{~m}$ & $255 \mathrm{~m}$ \\
\hline Région D ... & $11 \mathrm{~m}$. & $73 \mathrm{~m}$. & $18 \mathrm{~m}$. & $118 \mathrm{~m}$ & $36 \mathrm{~m}$ & $239 \mathrm{~m}$ \\
\hline Région $E$ & $6,65 \mathrm{~m}$. & $60,50 \mathrm{~m}$ & $10,35 \mathrm{~m}$ & $94 \mathrm{~m}$. & $19,8 \mathrm{~m}$. & $180,5 \mathrm{~m}$. \\
\hline Région $B$ & $1,57 \mathrm{~m}$ & $31,40 \mathrm{~m}$ & $2,45 \mathrm{~m}$ & $49 \mathrm{~m}$. & $4,86 \mathrm{~m}$ & $96 \mathrm{~m}$. \\
\hline
\end{tabular}

Ełude de l'évolution de l'amplitude sur le réel.

On a vu dans l'article précédent que l'on a

(15) . . n $=n_{0} \sqrt{\frac{U_{0}}{U}} \cdot \frac{1}{\left[\cdot l_{0}^{*} \beta d x\right]} \cdot \cos \frac{2 \pi}{V}(x-C T)$

En utilisant cette formule (15), commençons par examiner l'effet du facteur $U$, comme s'il existait seul. On aurait, pour le réel, une amplitude $n \quad(r, \infty)$ au large [c'est-à-dire pour $\left.\left(\frac{H}{\Lambda}\right) \supseteq 0,5\right]$; cette amplitude commencerait par diminuer au fur et à mesure que $\mathrm{H}$ diminue elle passerait par un minimum $n(r, A)$ dans la région $A$ avecn $(r, A)=0,90 \times n(r, \infty)$. L'amplitude croitrait alors si $\mathrm{H}$ continue à diminuer, pour passer parn $(r . D)=0,93 n(r, \infty)$ au point $D$, parn $(r, E)=n(r, \infty)$ au point $E$, par $\eta(r, B)=1,5 n(r, \infty)$ au point $B$, puis elle continuerait à croître jusqu'à ce que $n$ croissant pendant que $\mathrm{H}$ diminue, le rapport $\frac{\mathrm{n}}{\mathrm{H}}$ dépasse la valeur limite $\frac{n}{H}=\frac{1}{7}$, au-dessus de laquelle $\left(\frac{\mathrm{n}}{\mathrm{H}}\right)^{2} \geqslant\left(\frac{\mathrm{I}}{49}\right)$ n'est plus négligeable devant I'unité.

D'habitude, la région $A$ se trouve au voisinage de la passe du port ou en dehors de celui-ci. et l'amplituden $(r, \infty)$ a lieu pour des fonds $\mathrm{H}>50 \mathrm{~m}$., donc bien au large de la région étudiée. Pour cette raison, la faible décroissance de $n$ entre $n(r, \infty)$ et $n(r, A)$ n'est in connue, ni mesurée sur le réel.
Par contre, la croissance de $n$ entre les régions $A$ et $B$, puis après avoir dépassé $B$, se constate facilement sur les hauts-fonds par beau temps, lorsque le creux de la houle au large est très faible, pour que la houle ne déferle pas avant d'atteindre ces régions. La figure 5 ci-dessous donne 3 applications numériques de cette loi de variation de $n$ quand $H$ diminue, sous forme de trois courbes donnant $n$ en fonction de $\mathrm{H}$ respectivement dans les trois cas où $T=8 \mathrm{sec}$., $T=10 \mathrm{sec} ., T=14 \mathrm{sec}$.

On va maintenant tenir compte de la courbe de la figure 3 donnant $\beta \Lambda$, donc tenir compte des frottements. On voit que l'importance du facteur d'amortissement en $\left(\begin{array}{l}-j \beta d x \\ e\end{array}\right)$ est négligeable pour les profondeurs supérieures à celles de la zone $A$, car non seulement $(\beta \Lambda)$ est très faible, mais encore $\Lambda$ est grand, donc

$$
\beta=\frac{\beta \Lambda}{\Lambda} \text { est tout petit. }
$$

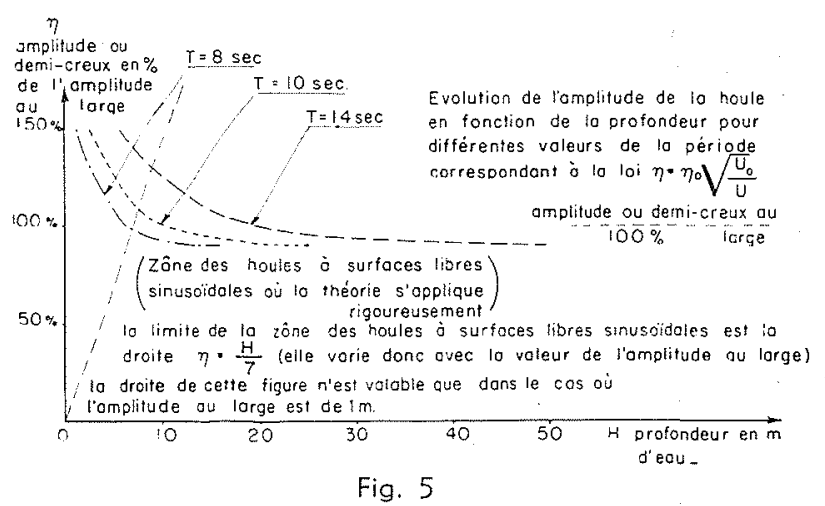


Dans les régions comprises entre $A$ et $B$, le facteur $\left(\begin{array}{l}-i \beta d x \\ e\end{array}\right)$ n'est plus négligeable, car non seulement $(\beta \Lambda)$ croît quand $H$ diminue, mais encore $A$ diminue, ce qui augmente $\beta=\frac{\beta \Lambda}{\Lambda}$. Si le chemin parcouru est assez long (comme c'est en général le cas), le facteur en $\beta \Lambda$ arrive à compenser et même à dépasser l'effet du facteur en $V U$.

Dans les régions de profondeurs plus faibles que $B$, le terme en $(-i \beta d x)$ est prépondérant, à moins que l'on ait affaire à de brusques ressauts du fond, produisant de véritables marches d'escalier qui ont alors un effet purement local. Ces régions sont en général inaccessibles aux navires (voir le tableau qui accompagne la figure 4 ).

Erude de l'évolution de l'amplisude n sur un modèle construith aves $\left(\frac{\alpha}{\theta^{2}}\right)=2$.

On a alors $\frac{h}{\lambda} \geq 2\left(\frac{H}{\Lambda}\right)$, l'inégalité étant d'autant plus marquée que $H$ est grand.

La région a de la maquette (voir figure 4) correspondant à la région $\mathrm{A}$ du modèle, se trouvera ò droite du point a sur la figure 4 . C'es: la région où se trouve en général le batteur qui engendre la houle.

La région e de la maquette correspondant i la région $E$ du réel, se trouve elle aussi déplacée vers la droite sur la figure 4.

Si on ne considère que le facteur en $\sqrt{u}$, on voit que l'amplitude $n(m)$ du modèle passe par son minimum entre a et $e$. Ceci aurait tendance à produire une agitation moindre dans le modèle que sur le réel, si on a réglé le batteur pour que les amplitudes en a et $A$ se correspondent. Mais le facteur en $\left(\begin{array}{l}-j \beta \mathrm{d} x \\ e\end{array}\right)$ serait moins important que sur le réel dans les cas où la maquette serait construite avec une échelle $\alpha$ telle que l'amortissement suive la même loi sur le modèle et sur le réel (voir article précédent).

On peut penser (sous réserve de vérification) que cette diminution d'importance du facteur en BA lqui équivaut à un gonflement de la houle par rapport à ce qu'elle serait si elle s'amortis. sait dans les mêmes conditions que sur le réel), compense la diminution relative de n entre le réel et le modèle, causée par le déplacement de A E en a e de l'arc de la courbe de U.
Les régions de profondeurs plus faibles que celles de la région $b$ peuvent exister au fond de certaines darses. Leur rôle amorrisseur sera beaucoup plus faible sur le modèle que sur le réel.

\section{3,32 - Conclusions relarives aux essais effec- tués sur une maqueřtre conservant unique- ment la similirude de formes.}

En définitive, l'évolution de l'amplitude de la houle ne sera pas rigoureusement identique sur le réel et sur le modèle, si on se borne à réaliser uniquement la similitude de formes en adoptant la distorsion maxima possible.

Cependant, sous réserve que l'échelle a soir telle que l'abaque d'où estrirée la figure $2 \mathrm{du}$ parag. 2,2 soit encore utillisable, on peut conclure que :

1. Les périodes de la houle incidente provo. quant des résonances avec les périodes propres des darses, se correspondront sensiblement suivant l'échelle $\theta$ des temps, si l'on se borne à explorer une gamme de périodes voisines de $T_{0}$, ce qui fait que l'essai sur modèle indiquera les périodes dangereuses de la houle incidente pour le port réel.

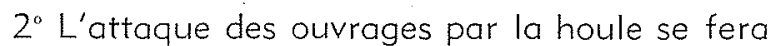
sous la même incidence sur le réel et sur le modèle; on pourra donc déterminer sur le modèle les emplacements optima des ouvrages amortisseurs à prévoir.

$3^{\circ}$ Les nœuds et les ventres des phénomènes stationnaires éventuels se produiront aux mêmes endroits sur le réel et sur le modèle.

$4^{\circ}$ Les rapports $N$ définis au sujet de l'étude de l'amortissement ne seront pas identiques sur le réel et sur le modèle; cependant, leurs valeurs seront probablement voisines les unes des autres, les valeurs de $N$ sur le modèle étant en général plus fortes que sur le réel, par suite de l'amortissement plus défectueux.

En définitive, une disposition de port jugée bonne sur le modèle sera certainement bonne sur le réel.

\section{CHAPITRE IV}

\section{EXPLICATION PROPOSEE POUR LES ACCIDENTS IMPROPREMENT APPELES "FLOTS DE FONDS " QUI ENDOMMAGENT LES « DIGUES MIXTES ».}

On adopte le terme de « digues mixtes» pour suivre la terminologie employée par $M$. I'Inspecteur général Watier dans son cours de Ports Maritimes, pour désigner les digues comportant une muraille verticale reposant sur le sol de fon- 
dation par l'intermédiaire d'un massif d'enrochements.

Lors des tempêtes exceptionnelles, la période de la houle peut atteindre 14 secondes environ. Le pied de la muraille verticale se trouvant a une cote comprise entre - 7 mètres et -11 mètres suivant les cas, le tableau de la page 29 montre que son point figuratif sur la courbe $\left(\frac{4 \pi}{g T}\right) \quad U=f\left(\frac{H}{\Lambda}\right)$ de la figure 3 se trouve, dans le cas exceptionnel, sur l'arc EB, au voisinage du point $B$, donc dans une région où la pente de cette courbe est très forte.

11 ne se produit rien en temps ordinaire, lorsque $T=8 \mathrm{sec}$. environ, car le point figuratif du pied de la muraille verticale se trouve alors au voisinage du point $D$. Dans le cas exceptionnel envisagé où $T \geqslant 14 \mathrm{sec}$. on voit que, si le chemin $x$ parcouru par les crêtes de vagues sur le massif d'enrochements (donc entre les fonds voisins de $-16 \mathrm{~m}$. et les fonds de $-8 \mathrm{~m}$. correspondant aux pieds de la muraille verticale) est très court, le gonflement des crêtes donné par la formule (15) est dû uniquement au facteur $\sqrt{\frac{U_{1}}{U}}$, le facteur $\left(\begin{array}{l}-i \beta d x \\ e\end{array}\right)$ n'ayant pas le temps de jouer. Dans ce cas non seulement l'amplitude $n$ de la houle se gonfle brusquement, par suite de la forte pente de l'arc EB de la courbe $\left(\frac{4 \pi}{g T}\right) \cup=f\left(\frac{H}{\Lambda}\right)$ (voir figure 3), mais encore la figure 6 montre que le rapport $\left(\frac{U}{c}\right)$ de la vitesse $U$ de propagation de l'éner. gie à la célérité de la crête, croît brusquement pendant le passage $\mathrm{DB}$ de la crête sur le mas.

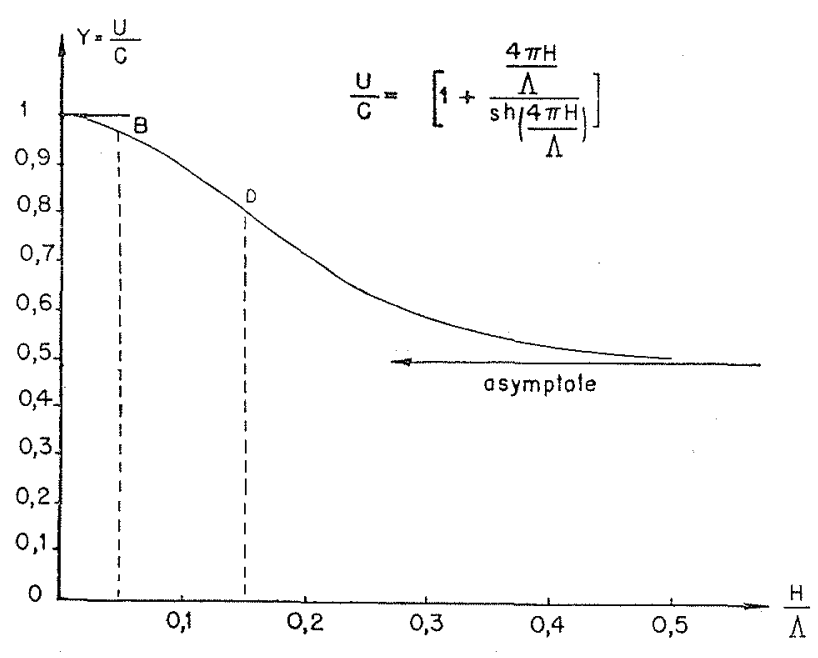

Fig. 6 sif d'enrochements, pour devenir voisin de l'unité lors du choc de la vague sur lo muraille verticale.

L'énergie apportée par chaque vague se propage donc, dans ce cas exceptionnel, avec la même vitesse que la crête, et la valeur de cette énergie est sensiblement

$$
E=\frac{1}{2} \circ g n^{2} \perp \text { par mètre courant de }
$$
crête.

Chacune de celles-ci est alors un formidable marteau d'eau qui provoque les dégâts consta. tés.

On voit aussi que le remède consiste à employer la branche montante de la courbe de $\beta A$ (voir figure 4) en faisant remonter le talus pour obtenir le profil classique de la digue à talus qui comporte un plan rugueux incliné à $3 / 1$ ou même à $5 / 1$ au-dessus du niveau - 3 mètres pour utiliser l'amortissement turbulent considérable qui correspond aux grandes valeurs de l'exposant $\beta \Lambda$ existant lorsque le paramètre $\mathrm{H}$ $\Lambda$ figure 4)

\section{CHAPITRE V}

\section{INFLUENCE DE LA DIRECTION DE LA HOULE INCIDENTE SUR LA FORMATION DE HOULES ET DE CLAPOTIS A GAUFRAGES RECTANGULAIRES.}

\section{Précision des mesures à effectuer sur le réel.}

Etant donné le fait que l'on devient plus exigeant qu'auparavant pour les caractéristiques de la maquette qui doivent assurer la correspondance des phénomènes du réel et du modèle, on doit être plus exigeant aussi pour la définition des conditions initiales de la houle, c'est-à-dire pour la définition de la houle au point repère (précédemment défini au sujet de la similitude de formes)

Jusqu'à présent, on s'est contenté, pour définir la direction de la houle incidente, des termes assez vagues utilisés par les marins : Nord, Sud, Es,t, Ouest, et même quelquefois Nord-Est, NordOuest, Sud-Est et Sud-Ouest. Cela revient implicitement à dire que I'on tolérait des erreurs inférieures ou égales à $22^{\prime \prime} 30^{\prime}$ sur la direction de propagation de la houle incidente. On va voir que l'étude de la houle gaufrée montre qu'il faut exiger une précision bien plus grande. 


\section{Etude de la houle gaufrée.}

Les résultats de l'étude systématique à la fois théorique et expérimentale des houles et clapotis gaufrés peuvent être résumés de la façon suivante :

Les surfaces libres de ces houles correspondent à l'équation :

$\eta=n_{n} \sin \frac{2 \pi}{\Lambda}(x-C t) \sin \left(\frac{2 \pi}{\Lambda^{1}} y\right)$

Elles sont caractérisées par l'existence de deux directions rectangulaires de lignes nodales, qui tracent ainsi des rectangles (voir figure 7 ).

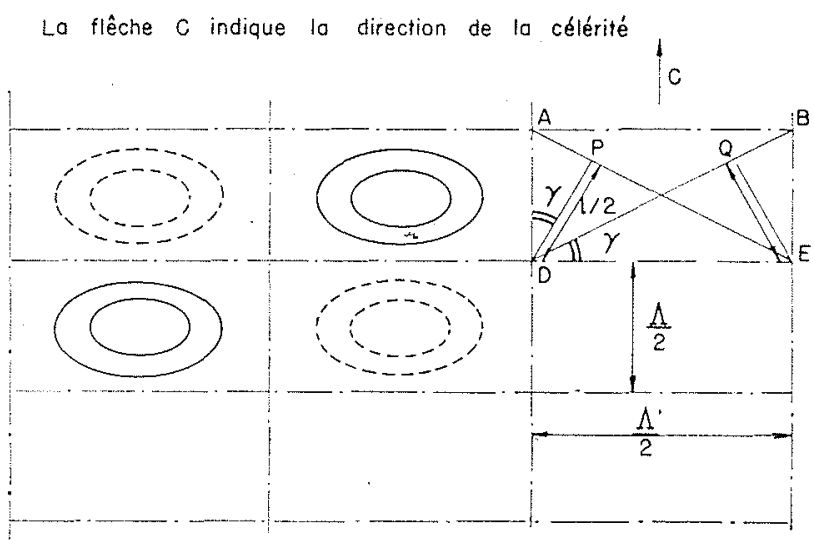

Fig. 7

L'une de ces directions est parallèle à la célérité $C$ de la surface ainsi définie. Ces houles peuvent être considérées comme résultant de l'addition des dénivellations de deux houles cylindriques composantes égales entre elles, d'amplitude $\frac{\eta_{11}}{2}$ et de longueur d'onde I. Les direc tions des crêtes de ces houles sont différentes; elles correspondent aux diagonales des rectan. gles dessinés par les lignes nodales.

On remarque que, contrairement à ce que I'on croit, d'habitude, la dénivellation résultante ne comporte aucun signe permettant à un œil non averti de déceler les houles cylindriques composantes.

$\mathrm{Si} H$ est la profondeur, $T$ la période et 2 y l'angle que forment entre elles les deux directions de crêtes $A E$, et $B D$ I'hydrody. namique classique irrotationnelle donne :

$$
\begin{aligned}
& \text { c.... }=\sqrt{\frac{g l}{2 \pi} \operatorname{Th}\left(\frac{2 \pi H}{l}\right)} \\
& \operatorname{avec}\left\{\begin{array}{l}
1=2 \overline{\mathrm{DP}}=2 \overline{\mathrm{EQ}} \\
\mathrm{c}=\left(\frac{1}{\mathrm{~T}}\right)
\end{array}\right.
\end{aligned}
$$

(célérité de la houle cylindrique composante).

$$
\begin{aligned}
& \text { On a d'autre part }: \Lambda=2 \overline{A D}=\frac{1}{\cos \gamma} \\
& \text { avec } \Lambda^{\prime}=2 \overline{A B}=\frac{1}{\sin \gamma} \\
& \text { On voit alors que }: \frac{\Lambda}{T}=c=\frac{c}{\cos \gamma} \\
& \text { avec } \frac{1}{\cos \gamma}=\sqrt{1+\left(\frac{\Lambda}{\Lambda^{\prime}}\right)^{2}}
\end{aligned}
$$

Cela donne :

$$
C=\sqrt{\frac{g \Lambda}{2 \pi} \sqrt{1+\left(\frac{\Lambda}{\Lambda^{\prime}}\right)^{2}} \text { Th }\left[\frac{2 \pi H}{\Lambda} \sqrt{1+\left(\frac{\Lambda}{\Lambda^{\prime}}\right)^{2}}\right]}
$$

On constate que cette formule est la généralisation dans le cas où la profondeur $\mathrm{H}$ est quelconque, de la formule donnée par le mathématicien anglais JEFFREY dans son étude de la houle gaufrée par profondeur infinie.

Les expériences faites dans un canal à profondeur constante $\mathrm{H}$ muni, à chacune de ses extrémités, d'un excellent amortisseur, à l'aide d'un volet générateur spécial dit «Volet souple transversal », vérifient cette formule pour un grand nombre de valeurs de $T=\frac{\Lambda}{\mathrm{C}}$ et de $\mathrm{H}$ correspondant à des valeurs de $\left(\frac{\Lambda}{\Lambda^{\prime}}\right)$ comprises entre 0.41 et 1.47 , avec des erreurs infé. rieures ou égales à $5 \%$ de la quantité mesurée. Ces expériences montrent également que la surface libre a bien la forme prévue, et que, conformément à la théorie, les plans verticaux parallèles à la direction de la célérité $C$ situés à mi-distance de deux lignes nodales, sont bien des «surfaces de courant», donc peuvent être adoptés comme limitantes solides sans perturber le phénomène.

On voit que, pour une profondeur $H$ donnée, il existe deux périodes (d'autant plus différentes que le rectangle est aplati) correspondant à un même quadrillage de lignes nodales; on obtient l'une ou l'autre période lorsque la célérité $C^{\prime}$ est parallèle à l'une ou l'autre des directions de lignes nodales.

Les clapotis gaufrés ont des surfaces libres de la forme $n=n_{n} \cos \left(\frac{2 \pi}{T} t\right) \sin \left(\frac{2 \pi}{\Lambda} x\right) \sin \left(\frac{2 \pi}{\Lambda^{\prime \prime}}\right)$

Ce sont des mouvements stationnaires.

Leur période est donnée par:

$$
T=\frac{\Lambda}{\sqrt{\frac{g \Lambda}{2 \pi} \sqrt{1+\left(\frac{\Lambda}{\Lambda^{\prime}}\right)^{2}} \text { Th }\left[\frac{2 \pi H}{\Lambda} \sqrt{1+\left(\frac{\Lambda}{\Lambda^{\prime}}\right)^{2}}\right]}}
$$


On voit que l'on a deux périodes différentes $T_{1}$ et $T_{2}$ quil sont toutes deux des périodes propres fondamentales pour une cuve parallélipipédique de dimensions $\frac{\Lambda}{2}, \frac{\Lambda^{\prime}}{2}$ et $H$. On obtient l'une ou l'autre de ces périodes fondamentales en permutant $A$ et $\Lambda$ ' dans la formule ci-dessus. Cela revient à faire tourner de $180^{\circ}$ une des houles composantes d'excitation du clapotis; l'autre houle composante d'excitation restant inchangée.

\section{Application aux ports}

Pour prendre un cas simple, considérons une darse de profondeur constante $H$ limitée par un quai $A$ B à muraille verticale. Supposons que ce quai soit attaqué par une houle cylindrique d'amplitude $n / 2$, de longueur d'onde 1 et de période $T$, dont les crêtes $a b$ forment un angle $y$ avec la normale $a n$ au quai $A B$ (voir figure 8 ).

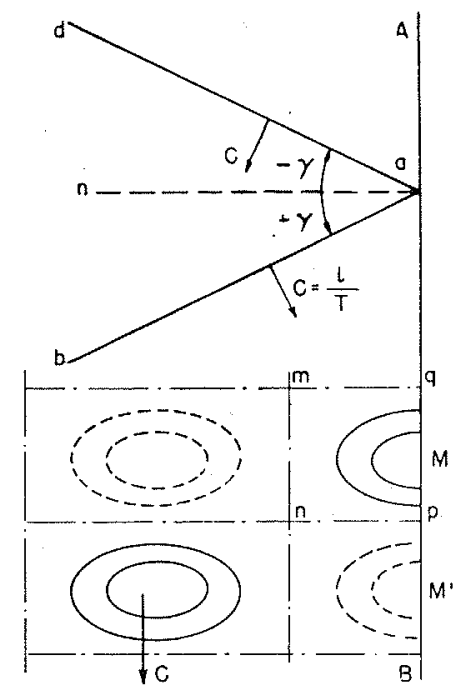

Fig. 8

Cette houle se réfléchit sur le quai $A B$ pour engendrer une seconde houle cylindrique d'amplitude $\frac{n}{2}$, de longueur d'onde 1 et de période $T$, mais dont les crêtes ont la direction a d faisant un angle - $y$ avec a $n$. La superposition de ces deux houles cylindriques donne une houle gaufrée rectangulaire d'amplitude $n$ dont le gaufrage $m \mathrm{n} \mathrm{p} \mathrm{q}$ est tel que non seulement sa célérité $C$ est parallèle au quai $A$, mais encore ce quai est une surface de courant de la houle, donc ne gêne nullement sa propagation. Cette dernière condition entraine le fait que les maxima $M$ et les minima $M^{\prime}$ de la dénivella. tion se produisent contre le quai $A$ B( 1 ).

Les longueurs des côtés du gaufrage de la houle $\mathrm{m} n \mathrm{p} \mathrm{q}$ dépendent non seulement de $\mathrm{T}$ et de $H$, mais encore de $\gamma$, puisque les deux longueurs d'onde de gaufrage sont :

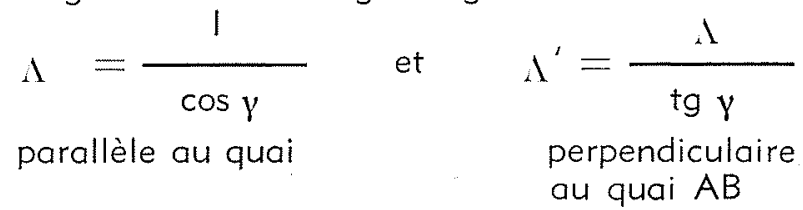

On en conclut que la forme du gaufrage de la houle résultante qui excite le plan d'eau de la darse dépend de l'angle $y$ d'incidence de la houle cylindrique perturbatrice, donc la forme de ce gaufrage dépend de l'orientation au point repère de la houle cylindrique arrivant du large.

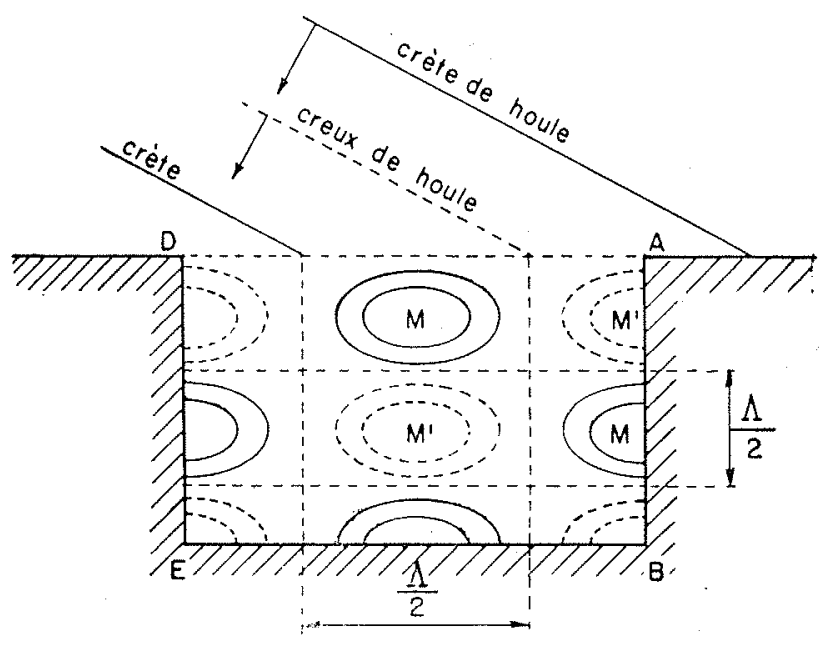

Fig. 9

(1) REMARQUE. - Certaines personnes ont émis l'idée que si le plan vertical imperméable $A B$ était remplacé por une discontinuité brusque du fond formant une "marche. d'escalier "donnant accès à un fond $\mathrm{H}^{\prime}$ situé beaucoup plus bas que le fond $\mathrm{H}$, on pourrait avoir une réflexion totale, sur $A B$, d'une houle cylindrique telle que $a b$, $a$ condition que l'angle $\gamma$ et les profondeurs $H$ et $H^{\prime}$ aient des valeurs appropriées.

Le raisonnement ci-dessus, valable dans les cas où $A B$ est une muraille verticale imperméable, montre que cette idée ne correspond pas à la réalité. En effet, la houle cylindrique de crête $a b$ a tendance à se réfléchir suivant $a d$ sur la «marche d'escalier " $A B$. Mais il se forme alors la houle gaufrée $m \mathrm{n} \mathrm{p} \mathrm{q}$, dont la célérité $C$ est parallèlé à $A B$ et dont les maxima et les minima $M$ et $M^{\prime}$ ont tendance à envahir la région située de l'autre côté de $A B$. L'expérience confirme cette dernière manière de voir. On constate en effet qu'une houle gaufrée $n$ se forme du côté de $A B$ où la profondeur est $H$, mais qu'une houle cylin. drique $\eta^{\prime}$ 'envahit également le plan d'eau situé du côté $A B$ où la profondeur est $H^{\prime}$. L'amplitude 1" ']' dépend des valeurs de $\mathrm{H}$ et de $\mathrm{H}^{\prime}$. On constate expérimentalement que la houle 1)' a changé de période, sa nouvelle période $T_{1}$ étant inférieure ou égale à la moitié de la période $T_{0}$ de la houle qui a provoqué la houle gaufrée 7 . Voir à ce sujet l'article de $M$. Carlotti sur la réflexion totale paru dans "La Houille Blanche », $N^{\circ} 2,1947$. 
Pour voir les effets susceptibles d'être produits par une variation de $\gamma$, commençons par considérer le cas simple où le quai $A B$ fait partie d'une darce rectangulaire $A$ B E D, de profondeur constante $\mathrm{H}$.

Les résonances se produisent entre la période de la houle gaufrée et les périodes propres de clapotis gaufrés des darses, lorsqu'il y a accord entre les $1 / 2$ longueurs d'ondes $\frac{\Lambda}{2}$ et $\frac{\Lambda^{\prime}}{2}$, et les dimensions linéaires $A B$ et $B E$ de la darse. Il en résulte que toutes les périodes propres de clapotis gaufrés de cette darse sont telles que: $A B=K\left(\frac{\Lambda}{2}\right)+\frac{\Lambda}{4}\left(\begin{array}{c}\text { nodale } A \\ \text { nod }\end{array}\right)$. $B E=K^{\prime}\left(\frac{\Lambda^{\prime}}{2}\right)$

$K$ et $K$ ' sont des nom̈bres entiers. Les résonances sont d'autant plus faciles à exciter que $K$ et $K^{\prime}$ sont petits.

On voit que, pour une valeur donnée de $T$, il existera quelques valeurs bien déterminées de $\gamma$ pouvant provoquer des résonances et qu'il n'y aura pas de résonance pour les autres valeurs de $\gamma$ :

En prenant le problème sous un autre aspect, on voit que la courbe de résonance Idonnant la valeur de l'amplitude des déplacements verticaux maxima de la surface libre en fonction de la période de la houle incidentel de la darse A B D E sous l'effet d'une houle cylindrique incidente $d^{\prime}$ orientation fixe $\gamma$, pour une gamme de période bien déterminée $T_{1}<T<T_{2}$ changera de forme suivant la valeur adoptée pour $Y$.

On en concluera facilement que, dans le cas de l'étude des rapports $\mathbf{N}$ d'un port, une variation de quelques degrés de l'orientation de la houle cylindrique incidente peut produire les mêmes effets qu'une variation de quelques secondes de la période de cette houle.

Pour faire des essais parfaits au point de vue théorique, il conviendrait donc de tracer non plus des courbes $N=f(T)$, mais des abaques $N=f(T, y)$.

Pour satisfaire ce besoin de précision dans la détermination des caractéristiques $T$ et $y$ de la houle incidente, les pays étrangers (Amérique et Angleterre) utilisent des relevés obtenus par photographies aériennes avec deux avions. Ces photos sont prises lorsque le soleil donne un éclairage rasant la surface de la mer, pour mettre en évidence les formes des crêtes de la houle cylindrique incidente. On photographie un chronomètre pour pouvoir connaittre l'heure exacte à laquelle chaque photo a été prise, pour en déduire la hauteur de la marée.

Pour compléter l'adaptation de la maquette au phénomène existant dans le port réel, on a pensé à faire un tarage, c'est-à-dire que l'on a pensé relever les déformations successives de la houle incidente au cours de son cheminement pour les reproduire sur la maquette. C'est une précaution excellente.

Cependant, comme l'étude de la maquette comportera l'étude des mouvements de la surface de l'eau dans les darses du port, il conviendrait de relever les caractéristiques de ce mouvement dans le port réel, dans les cas où l'on se propose d'étudier l'extension d'un port déjà existant

Le procédé qui consiste à utiliser des photos par l'éclairage rasant n'est plus applicable car, d'une part, les amplitudes des dénivellations à mettre en évidence sont très faibles; d'autre part, il s'agit de houles gaufrées qui se prêtent beaucoup plus mal que les houles cylindriques à la pratique des éclairages rasants.

La technique employée lors de l'étude de la forme de la surface libre des houles et des clapotis gaufrés en canal de laboratoire semble pouvoir se prêter à une généralisation sur les ports réels.

\section{Exposé rapide des résultats obtenus en canal}

Cas de la houle gaufrée. - On répand sur la surface de l'eau, de façon aussi uniforme que possible, des confettis blancs. On prend ensuite une photo en utilisant un appareil à plaque horizontale, dont l'axe optique est vertical, dont le temps de pose est légèrement inférieur à une période $T^{\prime}$ de la houle, et on manceuvre rapidement le diaphragme pour ramener l'ouverture de l'objectif de sa valeur maxima à sa valeur minima au cours du temps de pose.

L'épreuve ainsi obtenue présente de nombreuses virgules blanches dont la tête ronde se prolonge par une queue fine recourbée. En traçant les tangentes à l'origine de toutes ces virgules sur un calque, on obtient un champ de vecteur comme celui de la figure 10.

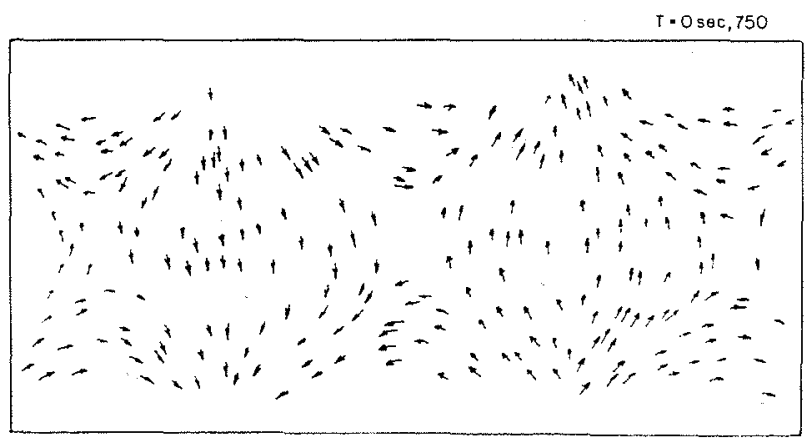

Fig. 10 
Cette figure permet de lire les valeurs de $\frac{\Lambda}{2}$ et $\frac{\Lambda^{\prime}}{2}$ avec une assez grande précision.

Cas des clapotis gaufrés. - On prend une photo analogue, sans prendre la peine de manœuvrer le diaphragme. On voit que, contrairement à ce qui se passait pour la photo prise sur la houle, la photo prise sur un clapotis permet de lire directement le tracé des lignes de courant de la surface libre. On peut voir également, sur la figure 11 que le tracé des lignes de courant est bien orthogonal à celui des lignes de niveau de la surface libre, comme l'indique la théorie des clapotis gaufrés.

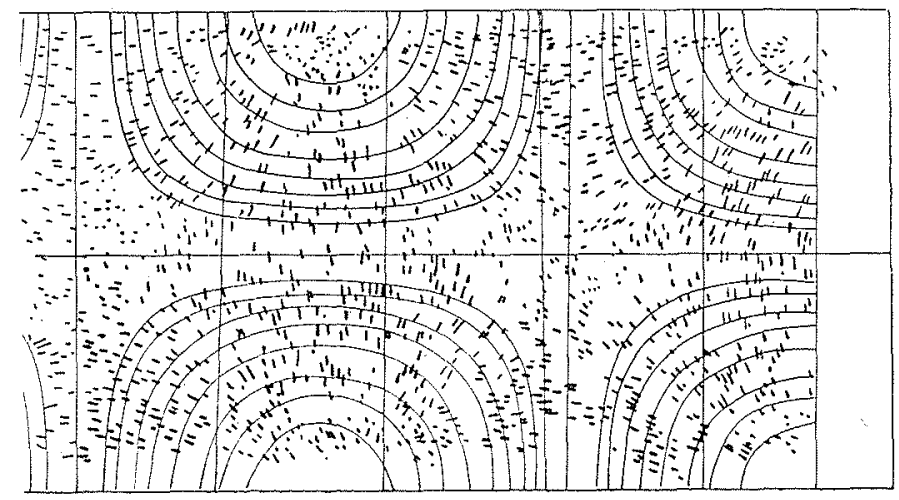

Fig. 11

On peut concevoir une généralisation de ce procédé pour l'étendue aux darses des ports réels, en prenant des photos aériennes de nuit avec de nombreux petits feux flottants (feux de bengale ou feu de magnésium). II ne faudra pas oublier de photographier en même temps un chronomètre pour connaître la hauteur de la marée.

\section{CHAPITRE VI}

\section{PRECISION DES MESURES A EFFECTUER SUR LE MODELE}

Les études expérimentales des clapotis gaufrés obtenus avec le «volet souple transversal » dans un canal parallélipipédique, ont montré que les courbes expérimentales de résonance donnant l'amplitude des maxima maximorum de la pente de la surface libre en fonction de la période $T$, du générateur, ressemblent à des cycloïdes, dont les points de rebroussement seraient tournés vers le haut. Ces courbes sont donc très pointues au voisinage des périodes propres de la cuve, c'està-dire dans les régions les plus intéresșantes.
Pour obtenir ces résultats, on a dû utiliser :

- un appareil optique indicateur de pente très sensible, sans inertie, et représenté sur la figure 12 ;

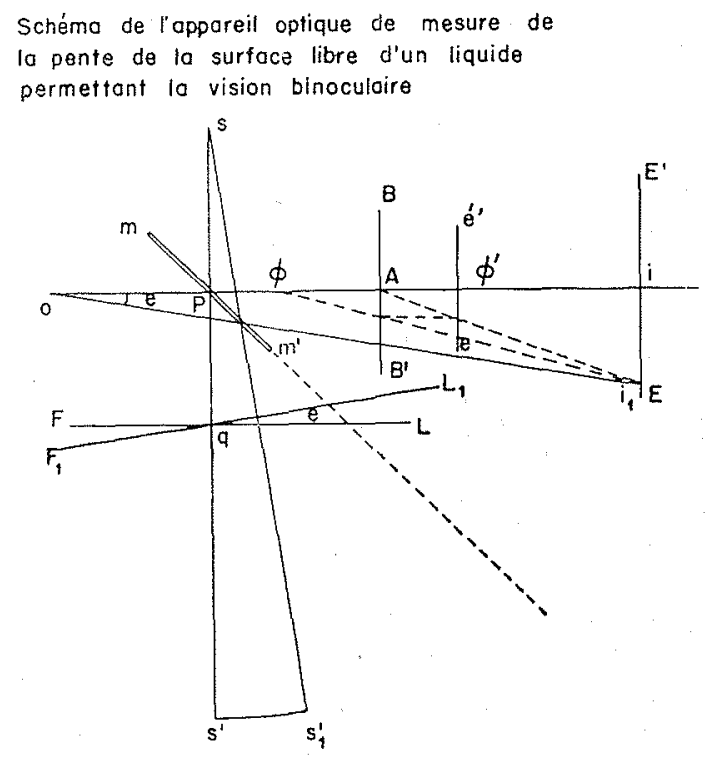

Fig. 12

- un moteur de batteur donnant une période constante, ou plutôt variant très lentement dans le temps, et tel que l'on connaisse la valeur de sa période avec une erreur de l'ordre de grandeur de un dix millième de $T$;

- un mode de mesure de cette période permettant la précision ci-dessus indiquée.

Ces précautions sont indispensables pour obtenir des expériences permettant de reproduire un relevé déjà fait et de retomber avec une erreur acceptable dans le voisinage immédiat du point représentant le relevé antérieur.

Pour relever une courbe de résonance de la maquette correspondant à :

- une valeur donnée $\gamma$ de la direction de la houle incidente;

- une hauteur donnée n de la houle au point repère mesurée par rapport au plan d'eau au repos;

- une gamme de périodes $T_{1}<T<T$, encadrant la valeur $T_{0}$ qui a servi à construire la maquette, on observera les conseils suivants:

On entrainera le volet avec un moteur électrique à courant continu à excitation séparée, alimenté par une batterie d'accus à grande capacité. On fera chauffer le moteur deux ou trois heures avani de commencer les mesures, pour réduire au minimum les variations de ses vitesses. On écrémera l'eau de la maquette tous les matins, et même deux fois par jour en été, les jours de vent. 
On place un excellent brise-lame amortisseur derrière le volet générateur.

Celui-ci devra être construit de manière telle qu'on puisse faire varier la direction de propagation de la houle incidente de quelques dizaines de degrés autour d'une direction principale.

Le pourtour du volet sera muni de joints en caoutchouc pour assurer l'étanchéité entre les deux cuves séparées par lui. Ces précautions, extrêmement utiles, ont pour but d'éviter la for. mation de noyaux tourbillonnaires engendrés par les tourbillons marginaux qui se produisent cux arêtes des plaques douées d'un mouvement normal à leur plan. Ces noyaux tourbillonnaires se dissipent lentement par viscosité et se promèneraient à tort et à travers dans la maquette.

Pour chaque valeur de $\mathrm{T}$ essayée, on réglera l'excentrique de commande du volet pour avorr la dénivellation $2 \eta_{\text {" }}$ voulue au point repère.

La valeur de la période $t$ du modèle sera mesurée en notant la durée de 100 tours consécutifs de la manivelle d'entraînement du volet. Cette mesure sera effectuée avec un chronomètre permettant de lire le $1 / 100^{\circ}$ de seconde. II existe des chronomètres dont l'aiguille trotteuse fait un tour toutes les 3 secondes, et permettant de lire facilement le $1 / 100^{\circ}$ de seconde. Ce chronomètre doit être mis en route et arrêté par une commande automatique branchée sur l'arbre moteur du volet.

\section{CHAPITRE VII}

\section{CONCLUSIONS}

La notion essentielle pour l'étude des mouve.ments de l'eau dans un port est la définition de la houle incidente par sa période $T$, par la direction $y$ de ses crêtes et par l'emplacement le plus voisin du rivage (déterminé par photographies aériennes) où elles sont encore rectrilignes, avant d'être déformées par l'influence des faibles profondeurs.

Du fait de l'existence de houles et de clapotis à gaufrages rectangulaires, pour lesquels les deux longueurs d'ondes longitudinale et transversale sont indépendantes l'une de l'autre, mais dépendent de l'action sur $T$ et $\gamma$ des limitantes solides des bassins de marées, un changement de la direction $\gamma$ de la houle incidente équivaut à un changement de sa période $T$ au point de vue des résonances possibles, avec les périodes propres des clapotis gaufrés des bassins de marée.
Si on considère un plan gradué en $T$ pour les abscisses et en $y$ pour les ordonnées, les états de la surface libre réellement possibles dans le port à étudier, correspondent à tous les points compris dans une certaine aire $A$ du plan $(T, \gamma)$.

Les états de la houle incidente créant une agitation critique dans un port formé de bassins de marée démunis d'ouvrages amortisseurs (quelle que soit la protection contre les houles incidentes) sont en nombre infini, car ils correspondent a tous les points formant un certain nombre de « courbes critiques » tracées sur le plan $(T y$ ). Pratiquement, il y aura toujours des ares de « courbes critiques » dans l'aire $A$, provoquanr des mouvements exagérés de la surface libre de l'eau du port. Le seul remède contre ces « états critiques » se trouve dans la mise en place, aux endroits adéquats, d'ouvrages amortisseurs bien étudiés qui diminuent l'amplitude des mouvements de claporis entretenus, correspondant a ces états critiques, en absorbant une partie notable de l'énergie contenue dans la faible houle d'excitation qui a réussi à entrer dans le port.

La détermination des emplacements d'ouvrages amortisseurs peut se faire avec précision à l'aide d'une maquette reproduisant exactement les évolutions des formes de crêtes dans le port réel. Certe reproduction a été appelée «similirude de formes $»$ dans le texte de la note.

On doit avoir recours à une distorsion des profondeurs pour éviter des maquettes trop étendues, à cause de la nécessité d'éviter des lames d'eau trop minces.

Cependant, pour obtenir la similitude de formes, ce n'est pas la distorsion que l'on a coutume de déduire de la loi de Reech-Froude qu'il faut adopter, car il n'y a pas une échelle des profondeurs, mais une fonction liant les profondeurs correspondantes du modèle et du réel. De plus, if existe une limite supérieure de la distorsion possible; il faudra donc, lorsquion construira une maquette, suivre la méthode indiquée dans la présente note.

On ajoute que le profil des ouvrages amortisseurs optima pour le port reel se détermine par des essais de l'ouvrage seul, construit sans distorsion et d̀ une échelle relativement grande, placé à une extrémité d'un canal à fond plat et à parois verticales parallèles, parcouru par une houle artificielle arrivant sur l'ouvrage.

Les premiers tracés de l'ouvrage sont établis en partant de tracés théoriques obtenus à l'aide des considérations exposées dans la présente note au sujet de l'amortissement de la houle.

Paris, le 27 mai 1946. 